\title{
LA FAMILIA HYDRAENIDAE MULSANT, 1844 (COLEOPTERA) EN LA PROVINCIA DE HUELVA (ESPAÑA). NUEVOS DATOS FAUNÍSTICOS (*)
}

\author{
Eva María Aceituno-Castro** y Carmen Elisa Sáinz-Cantero**
}

\section{Introducción}

Desde que Bigot y Marazanof (1966) citaran a Ochthebius (Aulacochthebius) exaratus Mulsant, 1844 y $O$. (Ochthebius) meridionalis Rey, 1885 en el Parque Nacional de Doñana, el conocimiento sobre los coleópteros Hydraenidae en Huelva se ha incrementado notablemente, siendo de destacar los trabajos de Montes et al. (1981), Jäch (1991, 1992), Garrido et al. (1996), Sáinz-Cantero et al. (en prensa) y Sáinz-Cantero \& Aceituno-Castro (en prensa), por haber sumado nuevos registros al catálogo provincial de coleópteros acuáticos.

Sin embargo es necesario puntualizar que la atención de éstos y otros autores se ha centrado exclusivamente en dos zonas concretas, las marismas del bajo Guadalquivir y la Sierra de Aracena, no existiendo datos correspondientes al resto del territorio onubense.

La lista faunística que presentamos a continuación cubre gran parte de esta laguna de conocimiento; es el resultado del estudio taxonómico de 2362 ejemplares de Hydraenidae que fueron capturados en diversas campañas de muestreo realizadas desde octubre de 1994 a septiembre de 1996. En total se han identificado 21 especies de las cuales 6 se citan por primera vez en la provincia de Huelva, elevando a 28 el número de hidrénidos con distribución en esta zona.

\section{Resultados}

Hydraena (Phothydraena) atrata Desbrochers des Loges, 1891

U.T.M: 29SPB6372, 9-X-1994, Ribera de Malagón, Cabezas Rubias, Huelva, 200 m, $10^{7}$ (Fig. 1).

Observaciones: Es la cita más meridional a nivel peninsular. Constituye el primer registro para la provincia de Huelva.

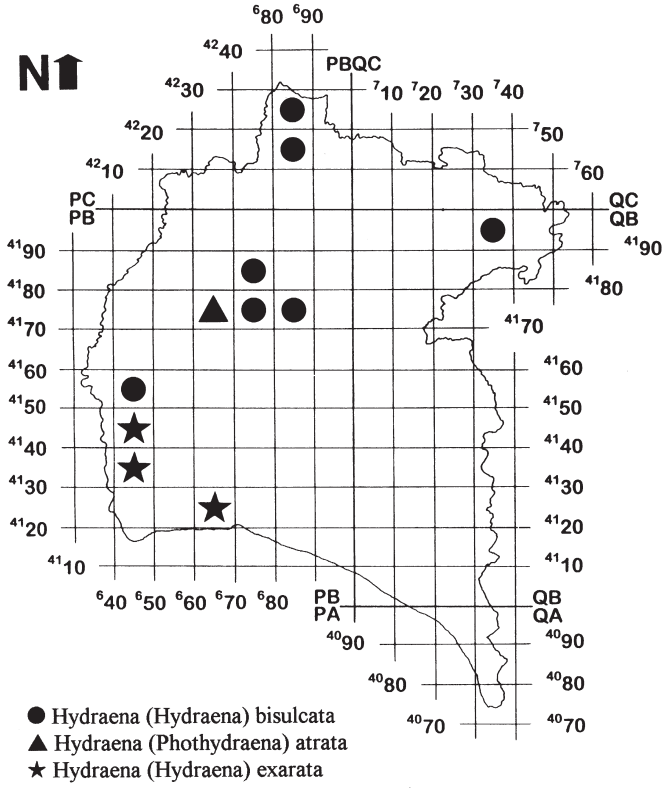

Fig. 1.- Nuevas citas de especies de Hydraenidae en la provincia de Huelva.

Fig. 1.- New records of Hydraenidae species in the Huelva province.

\section{Hydraena (Phothydraena) testacea Curtis, 1830}

U.T.M: 29SPB4776, 9-X-1994, Arroyo del Albahacar, Paymogo, Huelva, 150 m, 1 \%. U.T.M: 29SPB6372, 9-X-1994, Ribera de Malagón, Cabezas Rubias, Huelva, 200 m, 10". U.T.M: 29SPB8470, 11-XII-1994, Ribera de la Talliscas, Calañas, Huelva, 150 m, 1 ․ . U.T.M: 29SPB5367, 9-X-1994, Barranco de los Pilones, Puebla de Guzmán, Huelva, 150 m, 1 ㅇ (Fig. 2).

* Subvencionado por la DGICYT, proyecto FAUNA IBÉRICA - III, Nº PB92-0121.

** Departamento de Biología Animal y Ecología, Facultad de Ciencias, Universidad de Granada, 18071, Granada, España. 


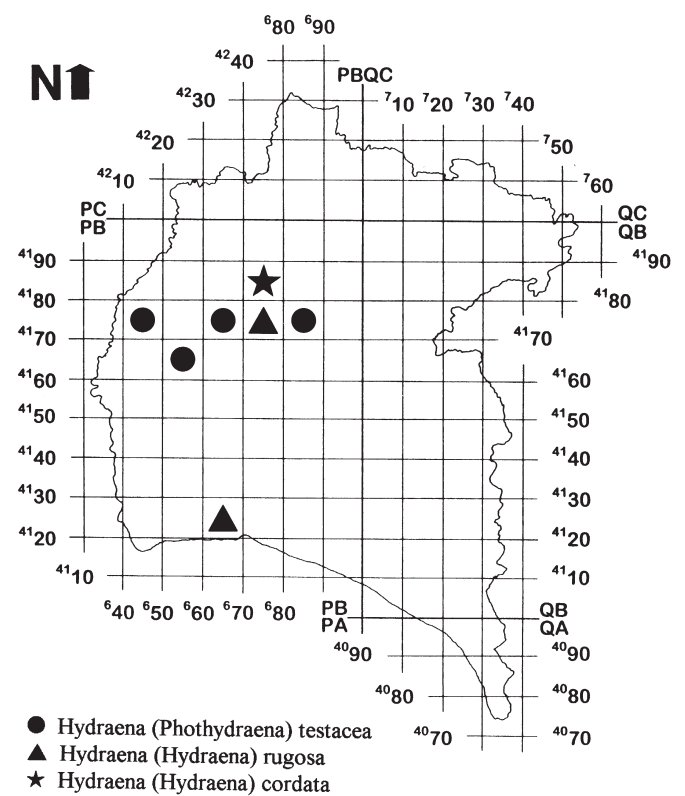

Fig. 2.- Nuevas citas de especies de Hydraenidae en la provincia de Huelva.

Fig. 2.- New records of Hydraenidae species in the Huelva province.

\section{Hydraena (Hydraena) bisulcata Rey, 1884}

U.T.M: 29SPC8222, 30-X-1994, Río Múrtiga, Encinasola, Huelva, 250 m, 10". U.T.M: 29SPC8718, 30-X-1994, Arroyo de Sillo, Cumbres de San Bartolomé, Huelva, $250 \mathrm{~m}, 20^{x} 0^{7}$ y 2 우 ㅇ. U.T.M: 29SQB3592, 8-XII-1994, Ribera de Huelva, Zufre, Huelva, $250 \mathrm{~m}, 40^{7} 0^{x}$ y 5 ㅇ + . U.T.M: 29SPB7180, 10 XII-1994, Ribera del Cañuelo, El Cerro de Andévalo, Huelva, 350 m, 1 ㅇ․ U.T.M: 29SPB7376, 11-XII-1994, Ribera de la Fresneda, El Cerro de Andévalo, Huelva, $200 \mathrm{~m}, 50^{7} 0^{7}$ y 6 오. U.T.M: 29SPB8470, 11-XII-1994, Ribera de la Talliscas, Calañas, Huelva, $150 \mathrm{~m}, 10^{\pi}$ y 2 우 ㅇ․ U.T.M 29SPB4452, 31-III-1996, Ribera de Tejada, El Granado, Huelva, 150 m, 1 ㅇ (Fig. 1).

Hydraena (Hydraena) cordata Schaufuss, 1833 U.T.M: 29SPB7180, 10-XII-1994, Ribera del Cañuelo, El Cerro de Andévalo, Huelva, 350 m, $40^{x} 0^{x}$ y 5 우 우 (Fig. 2).

\section{Hydraena (Hydraena) exarata Kiesenwetter, 1865}

U.T.M: 29SPB4742, 31-III-1996, Arroyo del Gico, Villanueva de los Castillejos, Huelva, 150 m, 2 \% . U.T.M: 29SPB4632, 31-III-1996, Arroyo del Alamillo, Villablanca, Huelva, $100 \mathrm{~m}$, 2 ㅇ ㅇ . U.T.M: 29SPB6823, 2-III-1996, Cañada de la Culata, Cartaya, Huelva, 40 m, $30^{\circ} \sigma^{\prime \prime}$ (Fig. 1).

Observaciones: Endemismo ibérico escasamente citado en el entorno peninsular. Se cita por primera vez en la provincia onubense.

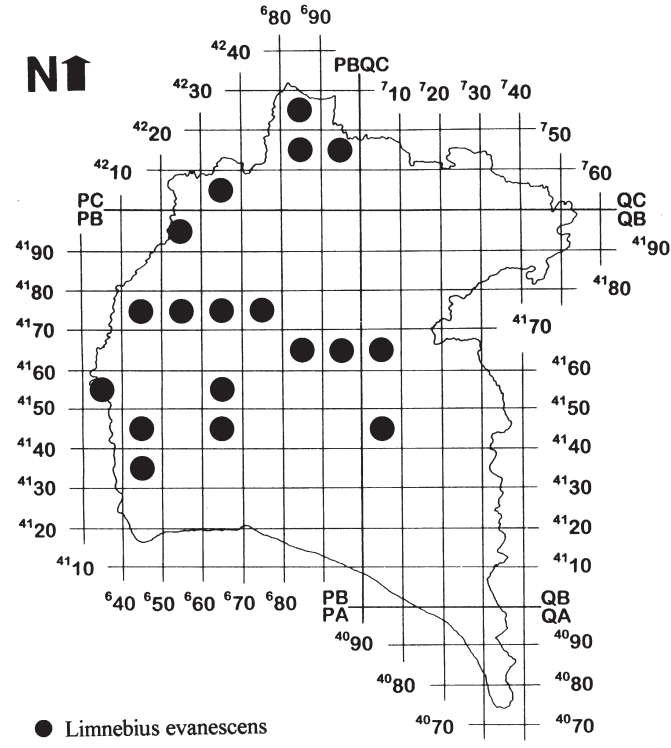

Fig. 3.- Nuevas citas de especies de Hydraenidae en la provincia de Huelva.

Fig. 3.- New records of Hydraenidae species in the Huelva province.

\section{Hydraena (Hydraena) rugosa Mulsant, 1844}

U.T.M: 29SPB7376, 11-XII-1994, Ribera de la Fresneda, El Cerro de Andévalo, Huelva, $200 \mathrm{~m}, 20^{7} \sigma^{7}$ y 4 우 우. U.T.M: 29SPB6823, 2-III-1996, Cañada de la Culata, Cartaya, Huelva, $40 \mathrm{~m}, 3$ 우 우 (Fig. 2).

Limnebius evanescens Kiesenwetter, 1865

U.T.M: 29SPC8222, 30-X-1994, Río Múrtiga, Encinasola, Huelva, $250 \mathrm{~m}, 40^{7} 0^{x}$ y 11 오. U.T.M: 29SPC8718, 30-X1994, Arroyo de Sillo, Cumbres de San Bartolomé, Huelva, $250 \mathrm{~m}, 10^{7}$ y 3 우 오. U.T.M: 29SPC9411, 29-X-1994, Río Múrtiga, Cumbres de San Bartolomé, Huelva, 300 m, $10^{7}$ y 1 ‥ U.T.M: 29SPC6204, 31-X-1994, Ribera de Chanza, Rosal de la Frontera, Huelva, $200 \mathrm{~m}, 40^{7} 0^{x}$ y $9 \%$ ․ U.T.M: 29SPB5898, 30-III-1996, Ribera de Calaboza, Rosal de la Frontera, Huelva, $150 \mathrm{~m}, 100^{\pi} 0^{\pi}$ y 28 우 ㅇ․ U.T.M: 29SPB4776, 9-X-1994, Arroyo del Albahacar, Paymogo, Huelva, $150 \mathrm{~m}, 170^{7} 0^{7}$ y 24 o $~$ ․ U.T.M: 29SPB5173, 9-X1994, Ribera de Malagón, Paymogo, Huelva, 100 m, 1560" $0^{7}$ y 193 우 ㅇ․ U.T.M: 29SPB6674, 9-X-1994, La Granadilla (Charca), Cabezas Rubias, Huelva, $150 \mathrm{~m}, 40^{7} \mathrm{o}^{7}$ y 2 \% 9. U.T.M: 29SPB6372, 9-X-1994, Ribera de Malagón, Cabezas Rubias, Huelva, $200 \mathrm{~m}, 30^{7} 0^{7}$ y 8 우 ㅇ. U.T.M: 29SPB7376, 11-XII-1994, Ribera de la Fresneda, El Cerro de Andévalo, Huelva, 200 m, 10". U.T.M: 29SPB8864, 14-IV-1995, Embalse del Calabazal, Calañas, Huelva, $200 \mathrm{~m}, 10^{7}$. U.T.M: 29SPB9260, 14-IV-1995, Arroyo Carrasco, Valverde del Camino, Huelva, $100 \mathrm{~m}, 20^{x} 0^{x}$ y 2 우 ㅇ. U.T.M: 29SQB0061, 13-IV-1995, Embalse de los Silillos, Valverde del Camino, Huelva, $200 \mathrm{~m}, 50^{7} 0^{x}$ y 4 ㅇ ․ U.T.M: 29SPB3753, 31-III- 


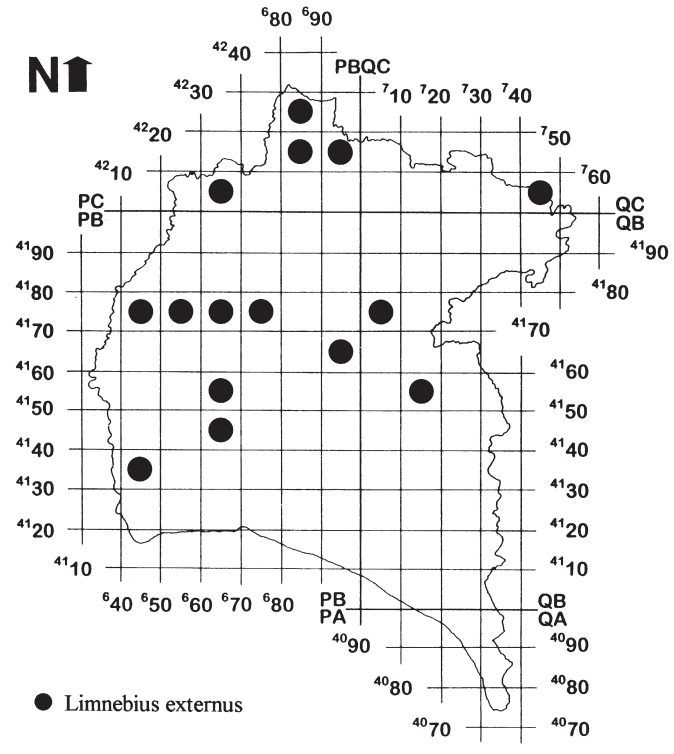

Fig. 4.- Nuevas citas de especies de Hydraenidae en la provincia de Huelva.

Fig. 4.- New records of Hydraenidae species in the Huelva province.

1996, Ribera de la Ronchona, El Granado, Huelva, 150 m, 1 ㅇ․ U.T.M: 29SPB6552, 15-IV-1995, Ribera del Aserrador, Villanueva de los Castillejos, Huelva, $150 \mathrm{~m}, 10^{7}$ y 1 ㅇ. U.T.M: 29SPB4742, 31-III-1996, Arroyo del Gico, Villanueva de los Castillejos, Huelva, $150 \mathrm{~m}, 20^{7} \sigma^{7}$ y 9 \% $ᄋ$. U.T.M: 29SPB5549, 15-IV-1995, Barranco del Guijo de María Martín, Villanueva de los Castillejos, Huelva, $100 \mathrm{~m}, 10^{\pi}$ y 2 우 ㅇ․ U.T.M: 29SQB0040, 13-IV-1995, Arroyo Candón, Beas, Huelva, $50 \mathrm{~m}, 20^{\pi} 0^{\pi}$ y 6 우 ㅇ․ U.T.M: 29SPB4632, 31-III-1996, Arroyo del Alamillo, Villablanca, Huelva, $100 \mathrm{~m}, 100^{x} \mathrm{o}^{x}$ y 17 우우 (Fig. 3).

\section{Limnebius externus Jäch, 1993}

U.T.M: 29SPC8222, 30-X-1994, Río Múrtiga, Encinasola, Huelva, $250 \mathrm{~m}, 150^{7} 0^{\pi}$ y 22 우 ․ U.T.M: 29SPC8718, 30-X1994, Arroyo de Sillo, Cumbres de San Bartolomé, Huelva, $250 \mathrm{~m}, 70^{7} 0^{7}$ y 3 우 ᄋ. U.T.M: 29SPC9411, 29-X-1994, Río Múrtiga, Cumbres de San Bartolomé, Huelva, 300 m, 1 ㅇ․ U.T.M: 29SPC6204, 31-X-1994, Ribera de Chanza, Rosal de la Frontera, Huelva, $200 \mathrm{~m}, 30^{x} \sigma^{x}$ y 9 \% ․ U.T.M: 29SQC4701, 10-X-1994, Ribera de Cala, Santa Olalla del Cala, Huelva, $450 \mathrm{~m}, 30^{7} \sigma^{\prime \prime}$ y 2 \% $~$ क . U.T.M: 29SPB4776, 9-X1994, Arroyo del Albahacar, Paymogo, Huelva, 150 m, 70 $0^{7}$ y 11 ㅇ ㅇ. U. U.T.M: 29SPB5173, 9-X-1994, Ribera de Malagón, Paymogo, Huelva, $100 \mathrm{~m}, 170^{7} \sigma^{7}$ y 10 \% . U.T.M: 29SPB6674, 9-X-1994, La Granadilla (Charca), Cabezas Rubias, Huelva, 150 m, $10^{7}$. U.T.M: 29SPB6372, 9-X-1994, Ribera de Malagón, Cabezas Rubias, Huelva, $200 \mathrm{~m}, 90^{7} 0^{7}$ y 17 우 ㅇ․ . U.T.M: 29SPB7376, 11-XII-1994, Ribera de la Fresneda, El Cerro de Andévalo, Huelva, $200 \mathrm{~m}, 20^{7} \sigma^{7}$ y 1 \% U.T.M: 29SQB1474, 11-X-1994, Embalse de la Marismilla,

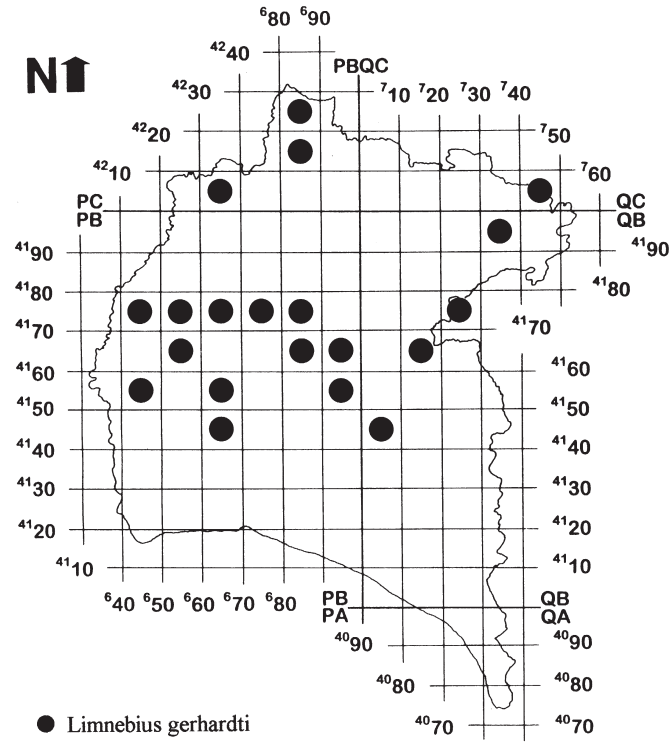

Fig. 5.- Nuevas citas de especies de Hydraenidae en la provincia de Huelva.

Fig. 5.- New records of Hydraenidae species in the Huelva province.

Nerva, Huelva, 300 m, $370^{\pi} \sigma^{\pi}$ y 47 q $q$. U.T.M: 29SPB9260, 14-IV-1995, Arroyo Carrasco, Valverde del Camino, Huelva, $100 \mathrm{~m}, 10^{x}$ y 5 우. U.T.M: 29SPB6552, 15-IV-1995, Ribera del Aserrador, Villanueva de los Castillejos, Huelva, $150 \mathrm{~m}$, 107. U.T.M: 29SQB1750, 19-III-1995, Arroyo Tamujoso, Paterna del Campo, Huelva, 100 m, $10^{7}$. U.T.M: 29SPB5549, 15-IV-1995, Barranco del Guijo de María Martín, Villanueva de los Castillejos, Huelva, $100 \mathrm{~m}, 2 \sigma^{7} \sigma^{7}$ y 2 \% $ᄋ$. U.T.M: 29SPB4632, 31-III-1996, Arroyo del Alamillo, Villablanca, Huelva, $100 \mathrm{~m}, 20^{7} \sigma^{7}$ (Fig. 4).

\section{Limnebius gerhardti Heyden, 1870}

U.T.M: 29SPC8222, 30-X-1994, Río Múrtiga, Encinasola, Huelva, $250 \mathrm{~m}, 50^{7} 0^{7}$ y 3 우 ㅇ. U.T.M: 29SPC $8718,30-\mathrm{X}-$ 1994, Arroyo de Sillo, Cumbres de San Bartolomé, Huelva, $250 \mathrm{~m}, 20^{\pi} \sigma^{\pi}$ y 2 우. ․ U.T.M: 29SPC6204, 31-X-1994, Ribera de Chanza, Rosal de la Frontera, Huelva, $200 \mathrm{~m}, 250^{x} 0^{x}$ y 35 ㅇ ㅇ․ . U.T.M: 29SQC4304, 1-XI-1994, Ribera de Cala, Santa Olalla del Cala, Huelva, $450 \mathrm{~m}, 50^{x} 0^{x}$ y 2 \% . U.T.M: 29SQB3592, 8-XII-1994, Ribera de Huelva, Zufre, Huelva, 250 m, 1․ ‥T.M: 29SPB4776, 9-X-1994, Arroyo del Albahacar, Paymogo, Huelva, $150 \mathrm{~m}, 30^{7} 0^{7}$ y 6 우 ․ U.T.M: 29SPB5173, 9-X-1994, Ribera de Malagón, Paymogo, Huelva, $100 \mathrm{~m}, 280^{\prime 7} \sigma^{7}$ у 28 ㅇ 우 . U.T.M: 29SPB6674, 9-X-1994, La Granadilla (Charca), Cabezas Rubias, Huelva, $150 \mathrm{~m}, 30^{7} \sigma^{7}$ y 2 우 ㅇ. U.T.M: 29SPB6372, 9-X-1994, Ribera de Malagón, Cabezas Rubias, Huelva, $200 \mathrm{~m}, 310^{x} 0^{x}$ y $28 \%$ $\uparrow$. U.T.M: 29SPB7376, 11-XII-1994, Ribera de la Fresneda, El Cerro de Andévalo, Huelva, 200 m, 10". U.T.M: 29SPB8470, 11-XII1994, Ribera de la Talliscas, Calañas, Huelva, $150 \mathrm{~m}, 20^{7} 0^{x}$ y 2 우 ㅇ. U.T.M: 29SQB2074, 8-X-1994, Ribera del Jarrama, 


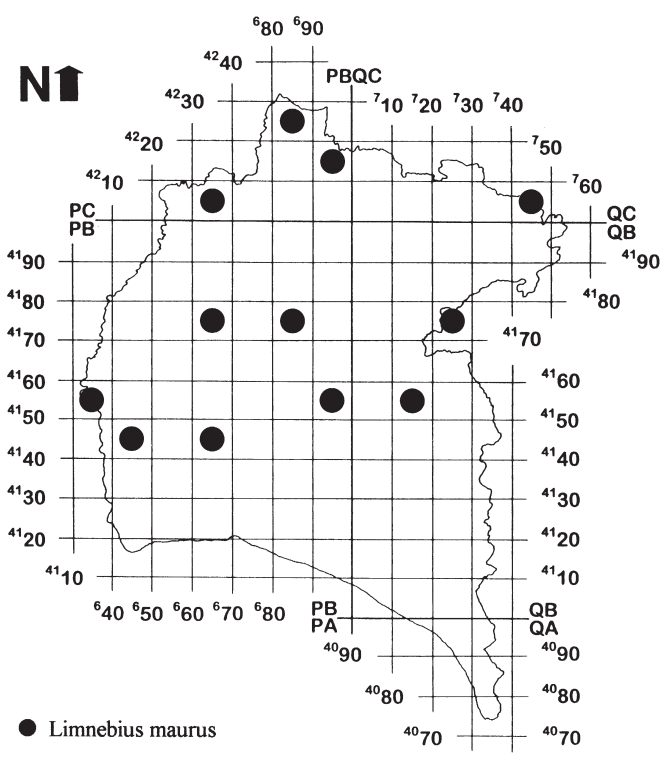

Fig. 6.- Nuevas citas de especies de Hydraenidae en la provincia de Huelva.

Fig. 6.- New records of Hydraenidae species in the Huelva province.

Nerva, Huelva, 250 m, $10^{\pi}$ y 2 ㅇ ㅇ. U.T.M: 29SPB5367, 9-X1994, Barranco de los Pilones, Puebla de Guzmán, Huelva, 150 m, 107. U.T.M: 29SPB8864, 14-IV-1995, Embalse del Calabazal, Calañas, Huelva, 200 m, 1 오. U.T.M: 29SPB9260, 14-IV-1995, Arroyo Carrasco, Valverde del Camino, Huelva, 100 m, 1ㅇ․ U.T.M: 29SQB1662, 8-X-1994, Arroyo las Telliscas, Berrocal, Huelva, 100 m, 1 ․ U.T.M: 29SPB4452, 31-III-1996, Ribera de Tejada, El Granado, Huelva, $150 \mathrm{~m}, 10^{7}$ y 1 ㅇ․ U.T.M: 29SPB6552, 15-IV-1995, Ribera del Aserrador, Villanueva de los Castillejos, Huelva, $150 \mathrm{~m}, 10^{x}$ y 1 우 U.T.M: 29SPB9351, 13-IV-1995, Arroyo de la Parrilla, Beas, Huelva, 200 m, $2 \sigma^{7} \sigma^{7}$ y 1 우. U.T.M: 29SPB5549, 15-IV-1995, Barranco del Guijo de María Martín, Villanueva de los Castillejos, Huelva, $100 \mathrm{~m}, 130^{x} 0^{\pi}$ y 7 우. U.T.M: 29SQB0040, 13-IV-1995, Arroyo Candón, Beas, Huelva, 50 m, $10^{7} \mathrm{y} 1$ 웅(Fig. 5).

\section{Limnebius maurus Balfour-Browne, 1978}

U.T.M: 29SPC8222, 30-X-1994, Río Múrtiga, Encinasola, Huelva, 250 m, 4 웅. U.T.M: 29SPC9411, 29-X-1994, Río Múrtiga, Cumbres de San Bartolomé, Huelva, $300 \mathrm{~m}, 10^{17}$ y 1 ‥ U.T.M: 29SPC6204, 31-X-1994, Ribera de Chanza, Rosal de la Frontera, Huelva, 200 m, $10^{7}$. U.T.M: 29SQC4304, 1-XI1994, Ribera de Cala, Santa Olalla del Cala, Huelva, $450 \mathrm{~m}$ 10". U.T.M: 29SPB6372, 9-X-1994, Ribera de Malagón, Cabezas Rubias, Huelva, 200 m, 10". U.T.M: 29SPB8470, 11XII-1994, Ribera de la Talliscas, Calañas, Huelva, $150 \mathrm{~m}$ $20^{7} 0^{7}$ y 2 우 ㅇ․ U.T.M: 29SQB2074, 8-X-1994, Ribera del Jarrama, Nerva, Huelva, 250 m, $10^{7}$. U.T.M: 29SPB3753, 31 III-1996, Ribera de la Ronchona, El Granado, Huelva, $150 \mathrm{~m}$, 1 ㅇ. U.T.M: 29SPB9351, 13-IV-1995, Arroyo de la Parrilla,

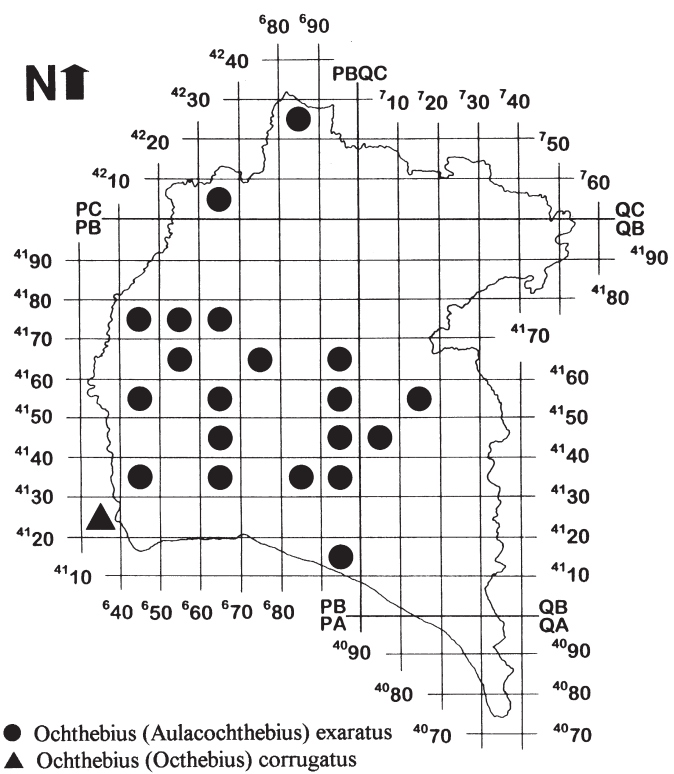

Fig. 7.- Nuevas citas de especies de Hydraenidae en la provincia de Huelva.

Fig. 7.- New records of Hydraenidae species in the Huelva province.

Beas, Huelva, 200 m, $30^{7} \sigma^{7}$. U.T.M: 29SQB1750, 19-III-1995, Arroyo Tamujoso, Paterna del Campo, Huelva, $100 \mathrm{~m}, 10^{\circ}$. U.T.M: 29SPB4742, 31-III-1996, Arroyo del Gico, Villanueva de los Castillejos, Huelva, 150 m, 2 우. . U.T.M: 29SPB5549, 15-IV-1995, Barranco del Guijo de María Martín, Villanueva de los Castillejos, Huelva, 100 m, $30^{x} 0^{x}$ y 2 우 우 (Fig. 6).

Ochthebius (Aulacochthebius) exaratus Mulsant, 1844

U.T.M: 29SPC8222, 30-X-1994, Río Múrtiga, Encinasola, Huelva, 250 m, 1 ㅇ. U.T.M: 29SPC6204, 31-X-1994, Ribera de Chanza, Rosal de la Frontera, Huelva, $200 \mathrm{~m}, 10^{\mathrm{T}}$ y 2 우 ㅇ․ U.T.M: 29SPB4776, 9-X-1994, Arroyo del Albahacar, Paymogo, Huelva, $150 \mathrm{~m}, 1240^{\lambda} \sigma^{\pi}$ y 131 우. U.T.M: 29SPB5173, 9-X-1994, Ribera de Malagón, Paymogo, Huelva, 100 m, 2 ㅇ ㅇ. U.T.M: 29SPB6674, 9-X-1994, La Granadilla (Charca), Cabezas Rubias, Huelva, 150 m, 1 ㅇ. U.T.M: 29SPB6372, 9-X-1994, Ribera de Malagón, Cabezas Rubias, Huelva, $200 \mathrm{~m}, 100^{7} 0^{\prime \prime}$ y 15 q ․ U.T.M: 29SPB5367, 9-X1994, Barranco de los Pilones, Puebla de Guzmán, Huelva, 150 m, $10^{7}$ y 1 ㅇ. U.T.M: 29SPB7465, 15-IV-1995, Arroyo Cascabelero, Villanueva de las Cruces, Huelva, $100 \mathrm{~m}, 1$ 오. U.T.M: 29SPB9260, 14-IV-1995, Arroyo Carrasco, Valverde del Camino, Huelva, 100 m, 1 ㅇ. U.T.M: 29SPB4452, 31-III1996, Ribera de Tejada, El Granado, Huelva, 150 m, 1 ㅇ. U.T.M: 29SPB6552, 15-IV-1995, Ribera del Aserrador, Villanueva de los Castillejos, Huelva, $150 \mathrm{~m}, 70^{7} \mathrm{o}^{7}$ y 7 오 오. U.T.M: 29SPB9351, 13-IV-1995, Arroyo de la Parrilla, Beas, Huelva, $200 \mathrm{~m}, 10^{7} 0^{7}$ y 2 \% $~$. U.T.M: 29SQB1750, 19-III1995, Arroyo Tamujoso, Paterna del Campo, Huelva, $100 \mathrm{~m}$, $110^{\pi} 0^{\pi}$ y 5 ㅇ ㅇ. U.T.M: 29SPB5549, 15-IV-1995, Barranco del Guijo de María Martín, Villanueva de los sCastillejos, Huelva, 


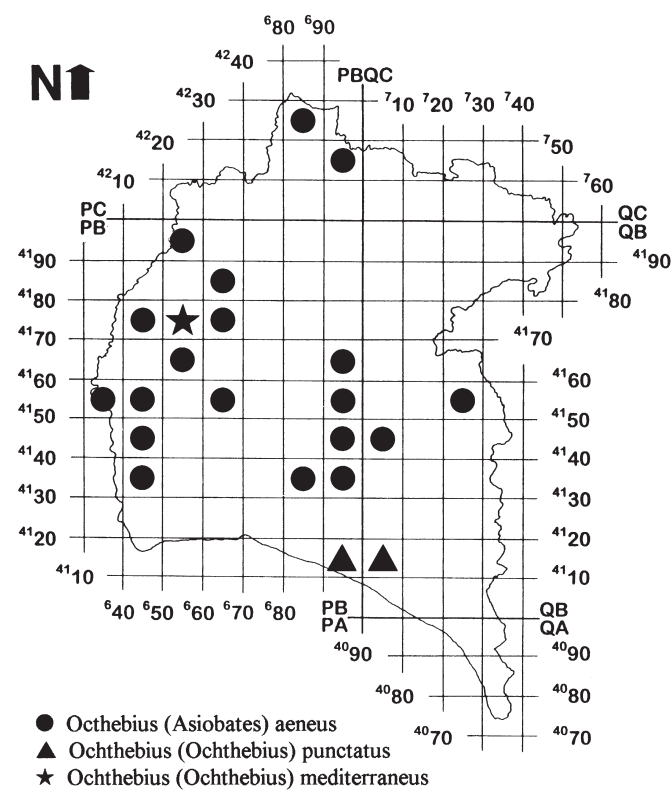

Fig. 8. - Nuevas citas de especies de Hydraenidae en la provincia de Huelva.

Fig. 8.- New records of Hydraenidae species in the Huelva province.

$100 \mathrm{~m}, 20^{\pi} \mathrm{o}^{\pi}$ y 1 ㅇ. U.T.M: 29SPB9244, 13-IV-1995, Arroyo de Pozo Ancho, Beas, Huelva, 50 m, 1 ㅇ. U.T.M: 29SQB0040, 13-IV-1995, Arroyo Candón, Beas, Huelva, $50 \mathrm{~m}, 20^{7} 0^{\prime \prime}$. U.T.M: 29SPB4632, 31-III-1996, Arroyo del Alamillo, Villablanca, Huelva, $100 \mathrm{~m}, 10^{7}$. U.T.M: 29SPB6330, 2-III1996, Arroyo Pilar de la Dehesa, Cartaya, Huelva, 40 m, $10^{7}$. U.T.M: 29SPB8038, 14-IV-1995, Arroyo del Tejar, Gibraleón, Huelva, $50 \mathrm{~m}, 1$ ㅇ. U.T.M: 29SPB9633, 19-III-1995, Arroyo del Pilar, San Juan del Puerto, Huelva, 0 m, $10^{x}$ y 2 우. U.T.M: 29SPB9115, 16-IV-1995, Arroyo Madre del Aviator, Moguer, Huelva, 0 m, $10^{\prime \prime}$ (Fig. 7).

\section{Ochthebius (Asiobates) aeneus Stephens, 1835}

U.T.M: 29SPC8222, 30-X-1994, Río Múrtiga, Encinasola Huelva, $250 \mathrm{~m}, 100^{7} \sigma^{7}$ y 9 우 우. U.T.M: 29SPC9411, 29-X1994, Río Múrtiga, Cumbres de San Bartolomé, Huelva, 300 m, $20^{\prime \prime} \sigma^{x}$. U.T.M: 29SPB5898, 30-III-1996, Ribera de Calaboza, Rosal de la Frontera, Huelva, 150 m, $10^{7}$. U.T.M: 29SPB6580, 10-XII-1994, Ribera de los Molinos, Cabezas Rubias, Huelva, 250 m, 1 \%. U.T.M: 29SPB4776, 9-X-1994, Arroyo del Albahacar, Paymogo, Huelva, $150 \mathrm{~m}, 310^{7} 0^{7}$ y 36 우 으. U.T.M: 29SPB6674, 9-X-1994, La Granadilla (Charca), Cabezas Rubias, Huelva, 150 m, 1 ․ U.T.M: 29SPB6372, 9-X-1994, Ribera de Malagón, Cabezas Rubias, Huelva, 200 m, $930^{7} 0^{\text {" }}$ y 69 우. U.T.M: 29SPB5367, 9-X1994, Barranco de los Pilones, Puebla de Guzmán, Huelva, $150 \mathrm{~m}, 30^{7} 0^{\pi}$. U.T.M: 29SPB9260, 14-IV-1995, Arroyo Carrasco, Valverde del Camino, Huelva, 100 m, 1 \%. U.T.M: 29SPB3753, 31-III-1996, Ribera de la Ronchona, El Granado, Huelva, 150 m, 1 ㅇ. U.T.M: 29SPB4452, 31-III1996, Ribera de Tejada, El Granado, Huelva, $150 \mathrm{~m}, 50^{7} \mathrm{o}^{7}$ y

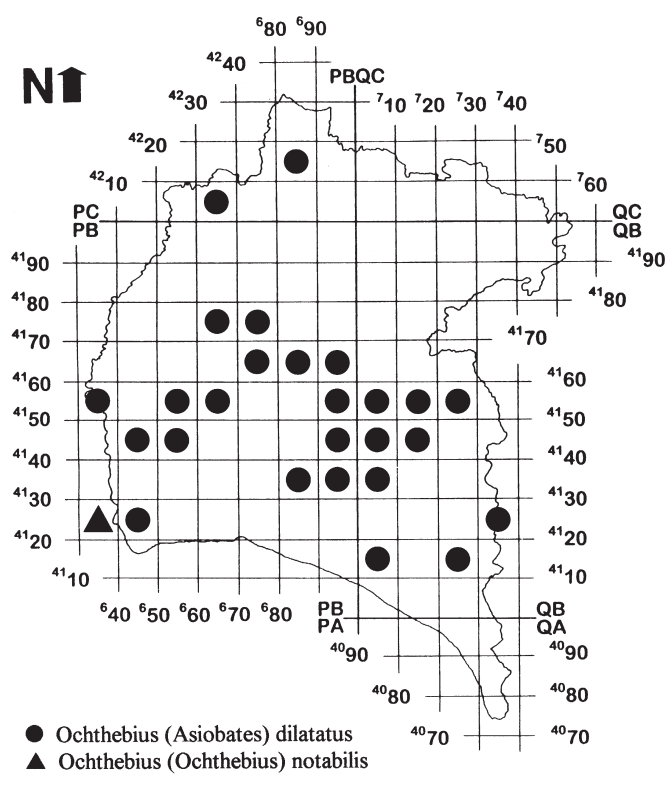

Fig. 9.- Nuevas citas de especies de Hydraenidae en la provincia de Huelva.

Fig. 9.- New records of Hydraenidae species in the Huelva province.

$20^{7} 0^{7}$. U.T.M: 29SPB6552, 15-IV-1995, Ribera del Aserrador, Villanueva de los Castillejos, Huelva, $150 \mathrm{~m}$, $30^{x} 0^{x}$ y 8 \% $q$. U.T.M: 29SPB9351, 13-IV-1995, Arroyo de la Parrilla, Beas, Huelva, $200 \mathrm{~m}, 260^{7} 0^{7}$ y 33 \% o. U.T.M: 29SQB2450, 18-III-1995, Río Corumbel, Paterna del Campo, Huelva, $100 \mathrm{~m}, 50^{7} 0^{7}$ y 10 우. U.T.M: 29SPB4742, 31-III1996, Arroyo del Gico, Villanueva de los Castillejos, Huelva, 150 m, 1 ․ . U.T.M: 29SPB9244, 13-IV-1995, Arroyo de Pozo Ancho, Beas, Huelva, $50 \mathrm{~m}, 20^{7} 0^{7}$ y 3 \% $\%$ U.T.M: 29SQB0040, 13-IV-1995, Arroyo Candón, Beas, Huelva, 50 m, $20^{\pi} 0^{\pi}$. U.T.M: 29SPB4632, 31-III-1996, Arroyo del Alamillo, Villablanca, Huelva, $100 \mathrm{~m}, 30^{7} 0^{7}$ y 3 o $~$. U.T.M: 29SPB8038, 14-IV-1995, Arroyo del Tejar, Gibraleón, Huelva, 50 m, $30^{7} 0^{7}$ y 1 ㅇ. U.T.M: 29SPB9633, 19-III-1995, Arroyo del Pilar, San Juan del Puerto, Huelva, 0 m, $400^{7} 0^{7}$ y 63 우 (Fig. 8).

\section{Ochthebius (Asiobates) dilatatus Stephens, 1829}

U.T.M: 29SPC8718, 30-X-1994, Arroyo de Sillo, Cumbres de San Bartolomé, Huelva, 250 m, $10^{7}$. U.T.M: 29SPC6204, 31-X-1994, Ribera de Chanza, Rosal de la Frontera, Huelva, 200 m, $20^{7}$ o $^{7}$. U.T.M: 29SPB6674, 9-X-1994, La Granadilla (Charca), Cabezas Rubias, Huelva, 150 m, 1 ㅇ․ U.T.M: 29SPB6372, 9-X-1994, Ribera de Malagón, Cabezas Rubias, Huelva, $200 \mathrm{~m}, 10^{7}$. U.T.M: 29SPB7376, 11-XII-1994, Ribera de la Fresneda, El Cerro de Andévalo, Huelva, $200 \mathrm{~m}$, 1 ㅇ. U.T.M: 29SPB7465, 15-IV-1995, Arroyo Cascabelero, Villanueva de las Cruces, Huelva, $100 \mathrm{~m}, 80^{7} 0^{7}$ y 7 우 오. U.T.M: 29SPB8864, 14-IV-1995, Embalse del Calabazal, Calañas, Huelva, $200 \mathrm{~m}, 10^{7}$. U.T.M. 29SPB9260, 14-IV1995, Arroyo Carrasco, Valverde del Camino, Huelva, 100 m, 


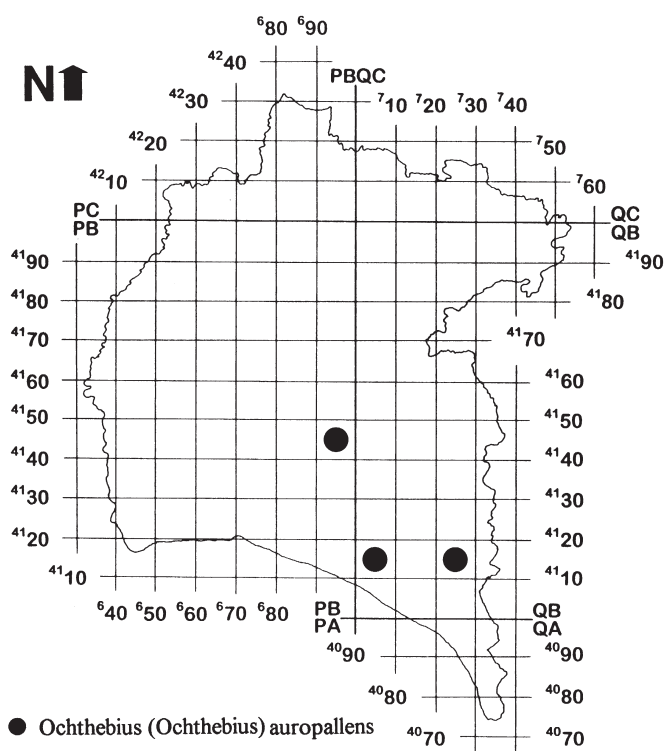

Fig. 10.- Nuevas citas de especies de Hydraenidae en la provincia de Huelva.

Fig. 10. - New records of Hydraenidae species in the Huelva province.

10". U.T.M: 29SPB3753, 31-III-1996, Ribera de la Ronchona, El Granado, Huelva, $150 \mathrm{~m}, 10^{\pi}$. U.T.M: 29SPB5455, 30-III-1996, Ribera de la Espada, El Almendro, Huelva, $250 \mathrm{~m}, 30^{7} 0^{7}$ y 1 \&. U.T.M: 29SPB6552, 15-IV1995, Ribera del Aserrador, Villanueva de los Castillejos, Huelva, $150 \mathrm{~m}, 20^{7} \sigma^{7}$ y 1 ㅇ. U.T.M: 29SPB9351, 13-IV1995, Arroyo de la Parrilla, Beas, Huelva, $200 \mathrm{~m}, 130^{x} \mathrm{o}^{x}$ y 4 우 ㅇ. U.T.M: 29SQB0751, 18-III-1995, Arroyo de la Soriana, Niebla, Huelva, $200 \mathrm{~m}, 60^{\prime \prime} 0^{\prime \prime}$ y 3 o ㅇ. U.T.M: 29SQB1750, 19-III-1995, Arroyo Tamujoso, Paterna del Campo, Huelva, $100 \mathrm{~m}, 90^{\circ} 0^{\text {八 y }} 16$ ㅇ ․ U.T.M: 29SQB2450, 18-III-1995, Río Corumbel, Paterna del Campo, Huelva, 100 m, 1 ㅇ․ U.T.M: 29SPB4742, 31-III-1996, Arroyo del Gico, Villanueva de los Castillejos, Huelva, $150 \mathrm{~m}, 10^{7}$ y 1 웅. U.T.M: 29SPB5149, 30-III-1996, Arroyo Montes, Villanueva de los Castillejos, Huelva, 200 m, 2 \% ․ . U.T.M: 29SPB9244, 13-IV-1995, Arroyo de Pozo Ancho, Beas, Huelva, 50 m, $10^{7}$. U.T.M: 29SQB0040, 13-IV-1995, Arroyo Candón, Beas, Huelva, $50 \mathrm{~m}, 20^{7} 0^{7}$ y 4 우. ‥T.M: 29SQB1543, 19-III1995, Arroyo Giraldo (Charca), La Palma del Condado, Huelva, $0 \mathrm{~m}, 120^{x} 0^{x}$ y 17 ㅇ ㅇ. U.T.M: 29SPB8038, 14-IV1995, Arroyo del Tejar, Gibraleón. Huelva, 50 m, $10^{x}$ y 2 우 우. U.T.M: 29SPB9633, 19-III-1995, Arroyo del Pilar, San Juan del Puerto, Huelva, 0 m, $110^{7} 0^{7}$ y 1 ㅇ. U.T.M: 29SQB0531, 3-III-1996, Arroyo Hondo, Bonares, Huelva, $100 \mathrm{~m}, 4$ 웅. U.T.M: 29SPB4628, 31-III-1996, Arroyo Pedraza, Villablanca, Huelva, 100 m, 1 ․ . U.T.M: 29SQB3028, 3-III1996. Arroyo de la Mayor, Hinojos, Huelva, $80 \mathrm{~m}, 10^{7}$ U.T.M: 29SQB0013, 16-IV-1995, Laguna Moguer II, Moguer, Huelva, 40 m, $50^{7} 0^{7}$ y 14 웅. U.T.M: 29SQB2412, 25-II-1995, Arroyo de Caño María, El Rocío, Huelva, 20 m, $20^{7} 0^{7}$ (Fig. 9).

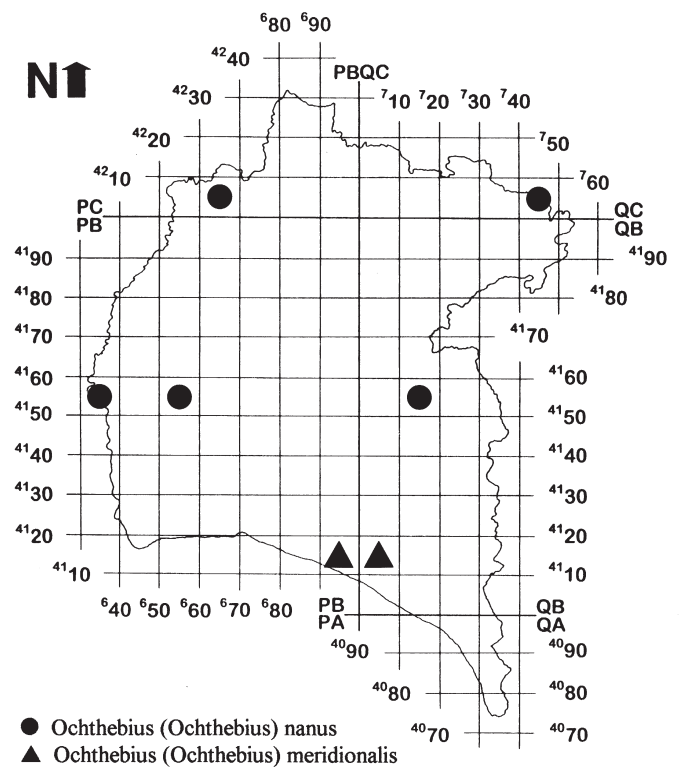

Fig. 11.- Nuevas citas de especies de Hydraenidae en la provincia de Huelva.

Fig. 11.- New records of Hydraenidae species in the Huelva province.

Ochthebius (Ochthebius) auropallens Fairmaire, 1879

U.T.M: 29SPB9244, 13-IV-1995, Arroyo de Pozo Ancho, Beas, Huelva, 50 m, 1 우. U.T.M: 29SQB0013, 16-IV-1995, Laguna Moguer II, Moguer, Huelva, $40 \mathrm{~m}, 20^{7} \sigma^{7}$. U.T.M: 29SQB2412, 25-II-1995, Arroyo de Caño María, El Rocío, Huelva, 20 m, $20^{7} \sigma^{\prime \prime}$ (Fig. 10).

Observaciones: Especie poco frecuente a nivel peninsular. Se señala por primera vez en la provincia de Huelva.

Ochthebius (Ochthebius) corrugatus Rosenhauer, 1856

U.T.M: 29SPB3923, 18-IX-1996, Estero de la Sardina (Los Regustos), Ayamonte, Huelva, $2 \mathrm{~m}, 200^{x} \sigma^{x}$ y 11 우 우 (Fig. 7).

Observaciones: Constituye una nueva cita para Huelva.

Ochthebius (Ochthebius) mediterraneus Ienistea, 1988

U.T.M: 29SPB5173, 9-X-1994, Ribera de Malagón, Paymogo, Huelva, 100 m, $20^{x} \sigma^{x}$ (Fig. 8)

Observaciones: Se cita por primera vez en la provincia de Huelva.

\section{Ochthebius (Ochthebius) meridionalis Rey, 1885}

U.T.M: 29SPB9115, 16-IV-1995, Arroyo Madre del Aviator (Marisma), Moguer, Huelva, 0 m, 107. U.T.M: 29SQB0013, 16-IV-1995, Laguna Moguer II, Moguer, Huelva, 40 m, $70^{7} 0^{x}$ y 11 우 우 (Fig. 11). 


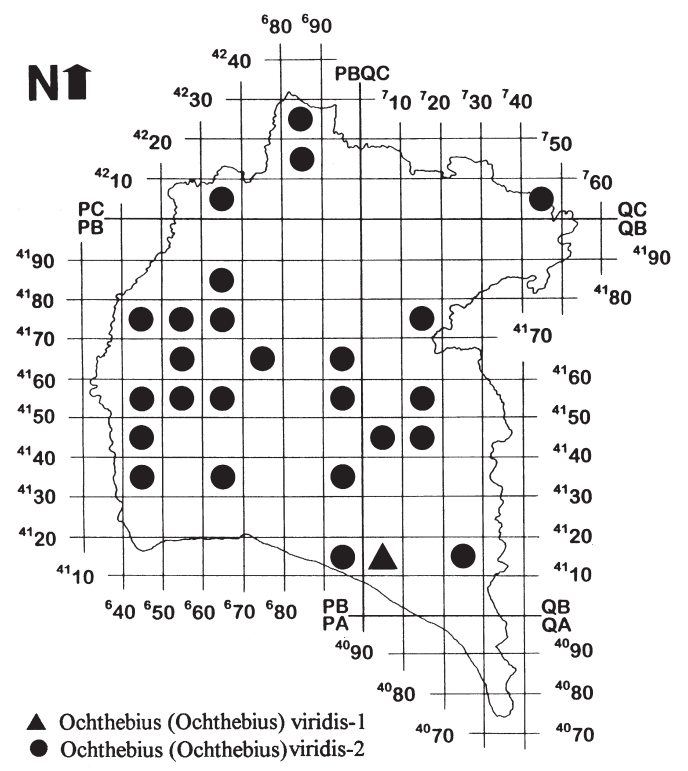

Fig. 12.- Nuevas citas de especies de Hydraenidae en la provincia de Huelva.

Fig. 12.- New records of Hydraenidae species in the Huelva province.

\section{Ochthebius (Ochthebius) nanus Stephens, 1829}

U.T.M: 29SPC6204, 31-X-1994, Ribera de Chanza, Rosal de la Frontera, Huelva, 200 m, $10^{7}$. U.T.M: 29SQC4701, 10-X1994, Ribera de Cala, Santa Olalla del Cala, Huelva, $450 \mathrm{~m}$, 2 우 ㅇ․ U.T.M: 29SPB3753, 31-III-1996, Ribera de la Ronchona, El Granado, Huelva, $150 \mathrm{~m}, 2$ 우. U.T.M: 29SPB5455, 30-III-1996, Ribera de la Espada, El Almendro, Huelva, 250 m, 1․ U.T.M: 29SQB1750, 19-III-1995, Arroyo Tamujoso, Paterna del Campo, Huelva, $100 \mathrm{~m}, 10^{\text {, }}$ (Fig. 11).

Ochthebius (Ochthebius) notabilis Rosenhauer, 1856 U.T.M: 29SPB3923, 18-IX-1996, Estero de la Sardina (Los Regustos), Ayamonte, Huelva, 2 m, $20^{x} 0^{x}$ (Fig. 9).

\section{Ochthebius (Ochthebius) punctatus Stephens, 1829}

U.T.M: 29SPB9115, 16-IV-1995, Arroyo Madre del Aviator (Marisma), Moguer, Huelva, 0 m, 2 우. U.T.M: 29SQB0013 16-IV-1995, Laguna Moguer II, Moguer, Huelva, 40 m, $20^{\pi} 0^{7}$ (Fig. 8).

Observaciones: Constituye la primera cita que se realiza para el territorio onubense.

Ochthebius (Ochthebius) viridis forma-1 Peyron, 1858 U.T.M: 29SQB0013, 16-IV-1995, Laguna Moguer II, Moguer, Huelva, 40 m, $80^{x} 0^{x}$ y 8 우 (Fig. 12).
Ochthebius (Ochthebius) viridis forma-2 Peyron, 1858

U.T.M: 29SPC8222, 30-X-1994, Río Múrtiga, Encinasola, Huelva, $250 \mathrm{~m}, 10^{7}$ y 1 ㅇ. U.T.M: 29SPC8718, 30-X-1994, Arroyo de Sillo, Cumbres de San Bartolomé, Huelva, $250 \mathrm{~m}$, $20^{x} 0^{\prime \prime}$. U.T.M: 29SPC6204, 31-X-1994, Ribera de Chanza, Rosal de la Frontera, Huelva, $200 \mathrm{~m}, 30^{7} 0^{7}$ y 1 \%. U.T.M: 29SQC4701, 10-X-1994, Ribera de Cala, Santa Olalla del Cala, Huelva, $450 \mathrm{~m}, 10^{7}$ y 1 ㅇ. U.T.M: 29SPB6580, 10-XII1994, Ribera de los Molinos, Cabezas Rubias, Huelva, 250 m, 1․ U.T.M: 29SPB4776, 9-X-1994, Arroyo del Albahacar, Paymogo, Huelva, $150 \mathrm{~m}, 220^{\circ} 0^{7}$ y 25 \& ․ U.T.M: 29SPB5173, 9-X-1994, Ribera de Malagón, Paymogo, Huelva, 100m, 10". U.T.M: 29SPB6674, 9-X-1994, La Granadilla (Charca), Cabezas Rubias, Huelva, $150 \mathrm{~m}, 80^{x} 0^{x}$ y 7 우. U.T.M: 29SPB6372, 9-X-1994, Ribera de Malagón, Huelva, $200 \mathrm{~m}, 220^{7} 0^{7}$ y 25 우. U.T.M: 29SQB1474, 11-X-1994, Embalse de la Marismilla, Nerva, Huelva, 300 m, $10^{7}$. U.T.M: 29SPB5367, 9-X-1994, Barranco de los Pilones, Puebla de Guzmán, Huelva, 150 m, 1 . U.T.M: 29SPB7465, 15-IV1995, Arroyo Cascabelero, Villanueva de las Cruces, Huelva, 100 m, 3 우 ㅇ. . U.T.M: 29SPB9260, 14-IV-1995, Arroyo Carrasco, Valverde del Camino, Huelva, $100 \mathrm{~m}, 20^{\circ} 0^{\circ}$ y 3 우 우. U.T.M: 29SPB4452, 31-III-1996, Ribera de Tejada, El Granado, Huelva, 150 m, $10^{x}$ y 1 ․ . U.T.M: 29SPB5455, 30III-1996, Ribera de la Espada, El Almendro, Huelva, $250 \mathrm{~m}$, 1 ㅇ. U.T.M: 29SPB6552, 15-IV-1995, Ribera del Aserrador, Villanueva de los Castillejos, Huelva, $150 \mathrm{~m}, 120^{7} \sigma^{7}$ y 6 웅. U.T.M: 29SPB9351, 13-IV-1995, Arroyo de la Parrilla, Beas, Huelva, 200 m, $10^{7}$. U.T.M: 29SQB1750, 19-III-1995, Arroyo Tamujoso, Paterna del Campo, Huelva, $100 \mathrm{~m}, 30^{7} 0^{71}$ y 3 우 0 . U.T.M: 29SPB4742, 31-III-1996, Arroyo del Gico, Villanueva de los Castillejos, Huelva, 150 m, 10". U.T.M: 29SQB0040, 13-IV-1995, Arroyo Candón, Beas, Huelva, $50 \mathrm{~m}, 70^{7} 0^{7}$ y 5 우 ㅇ․ . U.T.M: 29SQB1543, 19-III-1995, Arroyo Giraldo (Charca), La Palma del Condado, Huelva, 0 m, 2 ㅇ․ . U.T.M: 29SPB4632, 31-III-1996, Arroyo del Alamillo, Villablanca, Huelva, $100 \mathrm{~m}, 10^{7}$ y 2 우 우. U.T.M: 29SPB6330, 2-III-1996, Arroyo Pilar de la Dehesa, Cartaya, Huelva, 40 m, $10^{7}$. U.T.M: 29SPB9633, 19-III-1995, Arroyo del Pilar, San Juan del Puerto, Huelva, 0 m, 2 우. U.T.M: 29SPB9115, 16-IV-1995, Arroyo Madre del Aviator, Moguer, Huelva, 0 m, 1 ㅇ. U.T.M: 29SQB2412, 25-II-1995, Arroyo de Caño María, El Rocío, Huelva, 20 m, 1 우 (Fig. 12).

\section{Referencias}

Bigot, L. \& Marazanof, F., 1966. Notes sur l'ecologie des Coleóptères aquatiques des Marismas du Guadalquivir et premier inventaire des Coléoptères et Lépidoptères du Coto Doñana (Andalucía). Annls. Limnol., 2: 491-502.

Garrido, J., SÁinz-CANTERo, C.E. \& Díaz-PAzos, J.A., 1996. Fauna entomológica del Parque Nacional de Doñana (Huelva, España). I. (Coleoptera, Polyphaga). Nouv. Rev. Ent. (N.S.), Fasc. 1: 57-71.

JäCH, M.A., 1991. Revision of the Palearctic species of the genus Ochthebius Leach VI. The marinus group (Hydraenidae, Coleoptera). Entomologica Basiliensia, 14: 101-145.

JÄCH, M.A., 1992. Revision of the Palearctic species of the genus Ochthebius Leach, 1815. IX. The andraei and notabilis species groups (Coleoptera, Hydraenidae). NachrBl. Bayer. Ent., 41(1): 7-21. 
Montes, C., Muñoz-Valcarcel, F. \& Ramírez-Díaz, L., 1981. Estimas absolutas y relativas de la densidad de poblaciones de odonatos, coleópteros y heterópteros acuáticos en ecosistemas de nivel de agua fluctuante. Actas Cong. Esp. Limnol.: 51-60.

SÁinz-Cantero, C.E. \& Aceituno-Castro, E., (En prensa). Coleópteros acuáticos de Andalucía (España).I. Polífagos de la Sierra de Aracena (Huelva). (Coleoptera: Dryopidae, Elmidae, Helophoridae, Hydraenidae, Hydrochidae, Hydrophilidae). Elytron.
SÁinz-CANTero, C.E., Garrido, J. \& VAlladares, L.F., (En prensa). Los coleópteros Hydraenidae Mulsant,1844 de Andalucía (Sur de España): Nuevas aportaciones y análisis faunístico. Nouv. Rev. Ent.

Recibido, el 7-VII-1997 Aceptado, el 16-VII-1997 Publicado, el 31-X-1997 